\title{
Longitudinal Structure Function Using Thermodynamical Bag Model
}

\author{
S. Karthiyayini, K.K.Singh \\ Karthiyayini@bits-dubai.ac.ae; singh@bits-dubai.ac.ae \\ BITS Pilani Dubai Campus \\ Dubai International Academic City, Dubai, United Arab Emirate
}

\section{Abstract:}

A thermodynamical bag model is used to calculate the nucleon structure function by treating the quarks and gluons as Fermi and Bose gases. In this article this model is used to calculate the longitudinal structure function which is related to the ratio of absorption cross section for longitudinally and transversely polarized virtual photons. This can then be compared with the corresponding experimental values and other models. It is found to agree well with experimental data.

\section{INTRODUCTION:}

The deep inelastic cross section is expressed in terms of the nucleon structure functions. In the parton model of the nucleon, the deep inelastic scattering (DIS) cross section can be expressed as the incoherent sum of elastic lepton-quark cross sections, and hence it depends on the quark distribution functions. Thus the deep inelastic scattering experiments give valuable information on the quark distribution functions. The statistical model that is proposed for the nucleon yields the quark distribution functions that compare favorably well with the experimental results obtained from deep inelastic scattering experiments [1].

As it is the deep inelastic scattering of leptons that reveals much of the quark structure of the nucleon, let us start with a brief review of DIS. Then, following Devanathan et al. [1-5], we shall treat the nucleon as a MIT bag consisting of quarks and gluons. Treating them as Fermi gas and Bose gas and using their relevant statistical distribution functions, equations of state for the nucleon are obtained and solved self-consistently. Transformation of the Fermi and Bose statistical distribution functions to the infinite momentum frame yields the quark and gluon distribution functions. The nucleon structure functions are calculated using the quark distribution functions and compared with the results obtained from DIS experiments. If the spin degree of freedom is included in the Fermi statistical distribution function then one can obtain the quark spin distribution function and obtain the polarized nucleon structure functions. 


\section{The Statistical Model of the Nucleon}

The deep inelastic scattering of leptons on nucleons indicates that the nucleon consists of three valence quarks, sea quarks and gluons, confined within a small volume.

\begin{tabular}{|c|c|c|}
\hline Proton: & $\begin{array}{c}\text { u u d }+ \\
\text { (Valence quarks) }\end{array}$ & $\begin{array}{l}\text { quark-antiquark pairs + gluons } \\
\text { (Sea quarks) }\end{array}$ \\
\hline Neutron: & $\begin{array}{l}\text { : } u \text { d d }+ \\
\text { (Valence quarks) }\end{array}$ & $\begin{array}{c}\text { quark-antiquark pairs + gluons } \\
\text { (Sea quarks) }\end{array}$ \\
\hline
\end{tabular}

Based on this observation, a statistical model was developed for the nucleon. It is a MIT bag consisting of quark-gluon gas, for which the Fermi distribution function is used for describing the quarks and the Bose distribution function for describing the gluons. Treating the quarks as particles of zero rest mass, the number density of $u$-quarks with momentum lying between $\mathrm{p}$ and $\mathrm{p}+\mathrm{dp}$ at temperature $\mathrm{T}$ is given by the Fermi distribution function [2-7].

$$
n_{u}(p)=\frac{g}{(2 \pi)^{3}} \frac{1}{e^{\left(\epsilon-\mu_{u}\right) / T}+1}
$$

where $\epsilon$ is the energy and $\mu_{u}$ the chemical potential of the $u$-quark. The degeneracy factor $\mathrm{g}$ is 6 which is the number of degrees of freedom (3 due to colour and 2 due to spin) available for each flavour of quarks. Similar equations can be written for the $\mathrm{d}$-quarks and the antiquarks. The chemical potential for the $\mathrm{d}$-quark $\mu_{d}$ is, in general, different from that of $u$-quark. The chemical potential for the antiquark is of opposite sign to the chemical potential of the quark. With this observation, we can write down the distributions functions for $\mathrm{d}$-quarks and antiquarks.

$$
\begin{aligned}
& n_{d}(p)=\frac{6}{(2 \pi)^{3}} \frac{1}{e^{\left(\epsilon-\mu_{d}\right) / T}+1} \\
& n_{\bar{u}}(p)=\frac{g}{(2 \pi)^{3}} \frac{1}{e^{\left(\epsilon+\mu_{u}\right) / T}+1} \\
& n_{\bar{d}}(p)=\frac{g}{(2 \pi)^{3}} \frac{1}{e^{\left(\epsilon+\mu_{d}\right) / T}+1}
\end{aligned}
$$

Given the distribution functions, we can obtain the number density (number per unit volume) of each flavour of quarks by integration over the momentum.

$$
\begin{aligned}
& n_{u}=\int n_{u}(p) d^{3} p, \\
& n_{d}=\int n_{d}(p) d^{3} p,
\end{aligned}
$$




$$
\begin{aligned}
& n_{\bar{u}}=\int n_{\bar{u}}(p) d^{3} p, \\
& n_{\bar{d}}=\int n_{\bar{d}}(p) d^{3} p
\end{aligned}
$$

For the proton, the number of $u$ valence quarks is 2 and the number of $\mathrm{d}$ valence quarks is 1 . If $\mathrm{V}$ is the volume of proton, then

$$
n_{u}-n_{\bar{u}}=2 ; n_{d}-n_{\bar{d}}=1 \text {. }
$$

For the gluons, there is no number conservation and hence the chemical potential for the gluon is zero. The number density of the gluons is given by the Bose distribution function.

$$
n_{g}(p)=\frac{16}{(2 \pi)^{3}} \frac{1}{e^{\epsilon / T}-1}
$$

The degeneracy factor for the gluons is 16, of which 8 is due to the color degree of freedom and 2 due to the transverse components of spin.

In a similar way, one can find the energy density $\varepsilon_{q}$ of each flavor of quarks and antiquarks $(u, \mathrm{~d}$, $\bar{u}$ and $\bar{d}$ ) and calculate their contributions to the total energy density. As we have assumed zero rest mass for the quarks, the energy of the quark $\varepsilon$ is numerically equal to its momentum $\mathrm{p}$ in the natural units $(\hbar=c=1)$.

$\varepsilon_{q}=\int \frac{6}{(2 \pi)^{3}} \frac{p}{e^{\left(p-\mu_{q}\right) / T}+1} d^{3} p, \quad \varepsilon_{\bar{q}}=\int \frac{6}{(2 \pi)^{3}} \frac{p}{e^{(p+\mu q) / T}+1} d^{3} p$

For the gluons, the energy density is

$$
\varepsilon_{g}=\int \frac{16}{(2 \pi)^{3}} \frac{p}{e^{(p) / T}+1} d^{3} p
$$

The energy density due to all the quarks and gluons is the sum.

$$
\varepsilon=\varepsilon_{u}+\varepsilon_{d}+\varepsilon_{\bar{u}}+\varepsilon_{\bar{d}}+\varepsilon_{g}
$$

Now, the equations of state for the proton

$$
\begin{aligned}
& \varepsilon(T) V+B V=W \\
& n_{u}-n_{\bar{u}}=\frac{2}{V}=\mu_{u} T^{2}+\mu_{u}{ }^{3} / \pi^{2} \\
& n_{d}-n_{\bar{d}}=\frac{1}{V}=\mu_{d} T^{2}+\mu_{d}{ }^{3} / \pi^{2} \\
& P=\left(\frac{1}{3}\right) \varepsilon(T)-B=0
\end{aligned}
$$


The energy density $\varepsilon(T)$ is also a function of temperature. So, this bag model describes the nucleon not only in the ground state $(\mathrm{T}=0$ ) but also in the excited states at higher temperature. So, it can be truly described as the thermodynamical bag model of the nucleon. The bag constant is denoted by B and the volume of the bag by $\mathrm{V}$. The mass of the nucleon $\mathrm{M}$ in the thermodynamical bag model corresponds to $\mathrm{T}=0$ and $\mathrm{W}$ denotes the mass of the excited nucleon at some finite temperature $\mathrm{T}$. Equation (14) arises from the pressure balance condition or the energy minimization condition with respect to the bag volume.

Let us consider the ground state of the nucleon which corresponds to $\mathrm{T}=0$. Given the mass of the nucleon $\mathrm{M}=938.4 \mathrm{MeV}$, we can determine all the other four quantities by solving the four equations (11) - (14), using any numerical method such as the Newton-Raphson method.

$\mu_{u}=335.9 \mathrm{MeV}, \mu_{d}=266.6 \mathrm{MeV}, B^{\frac{1}{4}}=145.68 \mathrm{MeV}, \quad R=0.985 \mathrm{fm}$.

It is remarkable that this naive approach yields correctly the nucleon radius R. Assuming the value of the bag constant $\mathrm{B}$, one can determine $\mathrm{W} ; \mu_{u} ; \mu_{d} ; \mathrm{V}$ at any higher temperature by solving the equations of state. As it is possible to extend the study to higher temperatures by this method, this is known as the thermodynamical bag model (TBM). All parameters obtained so are tabulated in Table 1.

Table 1 Table showing the dependence of temperature $\mathrm{T}$, bag radius $\mathrm{R}$ and chemical potentials $\mu_{u} \longmapsto$ and $\mu_{d}$ on the Bjorken variable $\mathrm{x}$ along with the quark distribution functions for the proton in DIS $\left(\mathrm{Q}^{2}\right.$ $=4 \mathrm{Gev}^{2}$ )

\begin{tabular}{llllllllll}
$\mathrm{x}$ & $\mathrm{W}(\mathrm{MeV})$ & $\mathrm{T}(\mathrm{MeV})$ & $\mathrm{R}(\mathrm{fm})$ & $\mu_{u}(\mathrm{MeV})$ & $\mu_{\mathrm{d}}(\mathrm{MeV})$ & $\mathrm{x} u(\mathrm{x})$ & $\mathrm{x} d(\mathrm{x})$ & $\mathrm{x} \bar{u}$ & $\mathrm{x} \bar{d}$ \\
0.15 & 4854 & 85.7 & 2.1275 & 50.1 & 25.7 & 0.442 & 0.306 & 0.085 & 0130 \\
0.20 & 4110 & 85.5 & 2.0057 & 59.3 & 30.7 & 0.530 & 0.337 & 0.070 & 0.117 \\
0.30 & 3197 & 84.9 & 1.8297 & 76.7 & 40.6 & 0.559 & 0.301 & 0.035 & 0.069 \\
0.40 & 2623 & 84.2 & 1.6958 & 94.2 & 51.2 & 0.468 & 0.216 & 0.014 & 0.032 \\
0.50 & 2209 & 83.1 & 1.5805 & 113.2 & 63.5 & 0.345 & 0.136 & 0.005 & 0.012 \\
0.60 & 1883 & 81.6 & 1.4722 & 135.2 & 78.9 & 0.235 & 0.080 & 0.001 & 0.004 \\
0.70 & 1611 & 79.0 & 1.3622 & 162.6 & 100.0 & 0.153 & 0.043 & 0.000 & 0.001 \\
0.80 & 1371 & 74.0 & 1.2414 & 200.6 & 132.2 & 0.093 & 0.021 & 0.000 & 0.000 \\
0.90 & 1151 & 61.1 & 1.1025 & 259.4 & 187.6 & 0.042 & 0.006 & 0.000 & 0.000 \\
0.95 & 1045 & 45.9 & 1.0347 & 298.1 & 226.6 & 0.012 & 0.001 & 0.000 & 0.000 \\
1.00 & 938 & 0.0 & 0.9849 & 335.9 & 266.6 & 0.000 & 0.000 & 0.000 & 0.000 \\
\hline
\end{tabular}




\section{Longitudinal Structure function $\left(\mathrm{F}_{\mathrm{L}}\right)$}

Inclusive scattering cross section measurements are the important factor in understanding the nucleon structure in eN collision. The DIS cross section by virtual photon exchange is given by

$$
\frac{d^{2} \sigma}{d x d Q^{2}}=\frac{2 \pi \alpha^{2}}{Q^{4} x} Y_{+}\left(\left(F_{2}\left(x, Q^{2}\right)\right)-y^{2} F_{L}\left(x, Q^{2}\right)\right)
$$

where $Y_{+}=1+\left(1-y^{2}\right), y=Q^{2} / s x$ is the inelasticity, $\mathrm{s}$ is the center of mass energy squared and $\alpha$ is the fine structure constant. The structure functions $F_{2}$ and $F_{L}$ are related to the photon cross sections $\sigma_{L}$ and $\sigma_{T}$ as

$$
\begin{gathered}
F_{2}\left(x, Q^{2}\right)=\frac{Q^{2}}{4 \pi^{2} \alpha}\left(\sigma_{T}\left(x, Q^{2}\right)+\sigma_{L}\left(x, Q^{2}\right)\right) \\
F_{L}\left(x, Q^{2}\right)=\frac{Q^{2}}{4 \pi^{2} \alpha}\left(\sigma_{L}\left(x, Q^{2}\right)\right.
\end{gathered}
$$

Also, $0 \leq F_{L} \leq F_{2}$, because of the positivity of the cross sections.

The reduced cross section is defined as

$\sigma_{T}=F_{2}\left(x, Q^{2}\right)-\frac{y^{2}}{Y_{+}} \cdot F_{L}\left(x, Q^{2}\right)$

In Quark Parton Model, the photon interacts with quarks, the spin $1 / 2$ particle having only longitudinal momentum, i.e., the Callon - Gross relation. Therefore $F_{L}=0$. But in QCD, the quark interacts through gluon. Hence, the virtual photon also has transverse momentum and $F_{L}>0$. Due to its origin $F_{L}$ is directly connected with the gluon distribution in the proton and therefore can provide a sensitive test of perturbative QCD.

\section{RESULTS AND DISCUSSION:}

At finite energies the structure functions is replaced by the longitudinal structure functions

$$
F_{L}\left(x, Q^{2}\right)=F_{2}\left(x, Q^{2}\right)\left[1+\frac{4 M^{2} x}{Q^{2}}\right]-2 F_{1}\left(x, Q^{2}\right)
$$

which is in turn related to the ratio of absorption cross section for longitudinally and transversely polarized virtual photons

$$
R=\frac{\sigma_{L}}{\sigma_{T}}=\frac{F_{L}}{2 x F_{1}}
$$




\begin{tabular}{|l|l|l|l|}
\hline $\mathrm{X}$ & \multicolumn{1}{|c|}{$F_{2}$} & \multicolumn{1}{c|}{$F_{L}$} & \multicolumn{1}{c|}{$\mathrm{R}$} \\
\hline 0.15 & 0.2827 & -0.2127 & -0.7523 \\
\hline 0.2 & 0.3171 & -0.1298 & -0.4137 \\
\hline 0.3 & 0.3051 & 0.0754 & 0.2471 \\
\hline 0.4 & 0.2418 & 0.1808 & 0.7477 \\
\hline 0.5 & 0.1720 & 0.1886 & 1.0965 \\
\hline 0.6 & 0.1142 & 0.1528 & 1.3380 \\
\hline 0.7 & 0.0729 & 0.1102 & 1.5116 \\
\hline 0.8 & 0.0437 & 0.0720 & 1.6475 \\
\hline 0.9 & 0.0193 & 0.0342 & 1.7720 \\
\hline 0.95 & 0.0054 & 0.0100 & 1.8518 \\
\hline 1.0 & 0 & 0 & 0 \\
\hline
\end{tabular}

HERA as opened the field of low $\mathrm{x}$ physics which is governed by gluon interaction and which is far from being fully understood. The gluon momentum density at low $\mathrm{x}$ is very large. This causes the structure function $\mathrm{F}_{2}$ to rise at low $\mathrm{x}$. It determines the longitudinal structure function to be large and the production cross section of heavy flavors to be sizeable.

As expected even with our calculation it is found that $F_{L}$ is found to be larger than $F_{2}$. It is mainly due to the gluon emission as predicted earlier [8]. However although theoretical calculation predict a negative value for low $\mathrm{Q}^{2}$ region as the result got by our model too, a negative value of $F_{L}$ may not be measured. Experiments does not support the negative value of $F_{L}$. Here we have the limitation of our model. Hence the $\mathrm{x}$ dependence of it at low $\mathrm{Q}^{2}$ region needs to be studied thoroughly. Also more experimental data in this region may throw more light in this structure. It is proposed to further probe into the theory and a complete analysis to be done with our model.

Although the results of our model matches with the experimental data given below for the value of $\mathrm{x}$ in the range given in the table. 


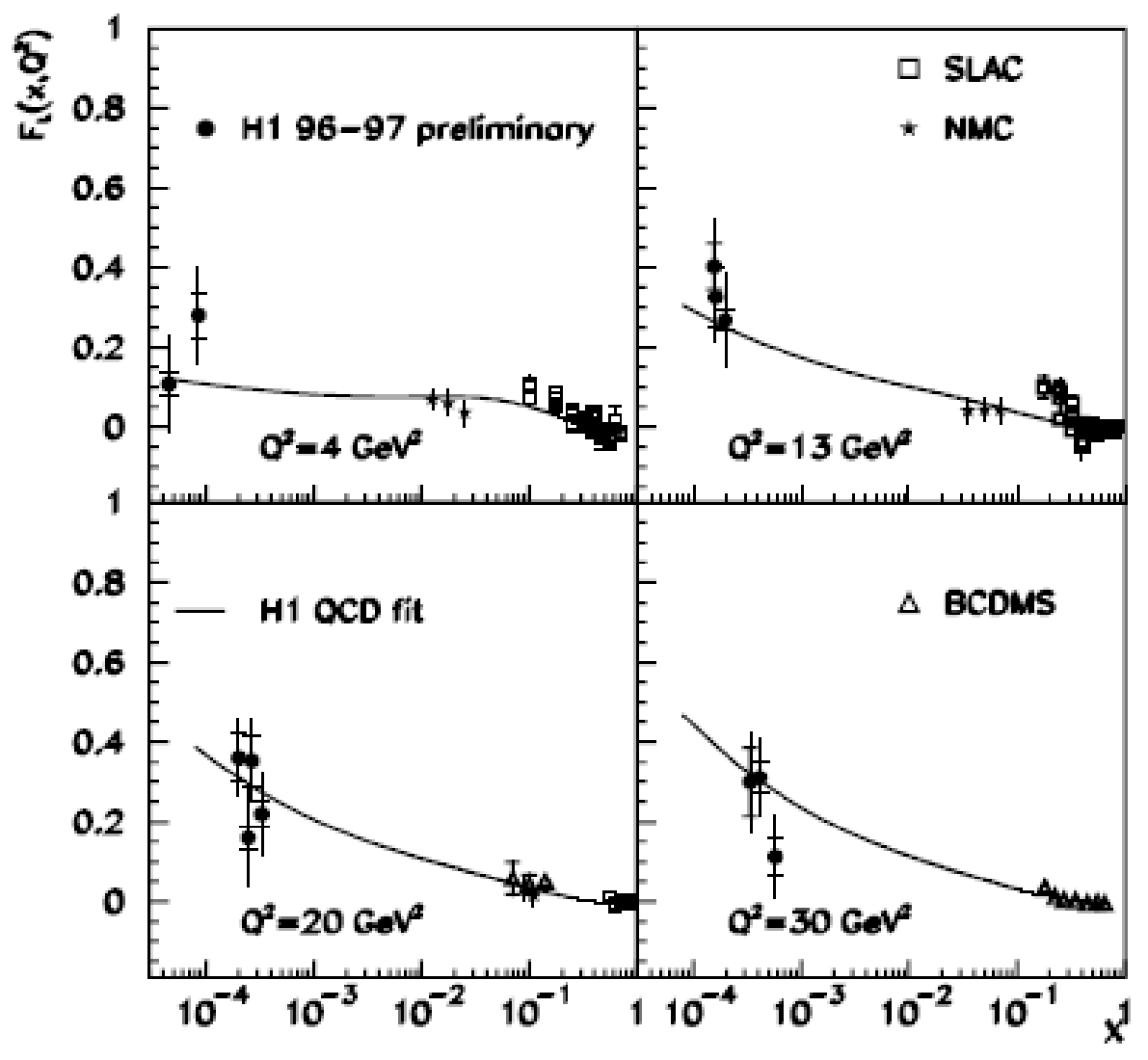

\section{REFERENCES:}

[1] V. Devanathan, S. Karthiyayini "The Legacy of Alladi Ramakrishnan in the Mathematical Sciences" Eds. Krishnaswami Alladi, J.R. Klauder and C.R. Rao, Springer (2010) 441 - 463.

[2] V. Devanathan, S. Karthiyayini and K. Ganesamurthy, Mod. Phys. Lett. A9, 3455 (1994)

[3] V. Devanathan, S. Karthiyayini and K. Ganesamurthy, Parton Distributions and Nucleon Structure Functions, in Perspectives in Theoretical Nuclear Physics (p.119), Eds. K. Srinivasa Rao and L. Satpathy, Wiley Eastern, New York (1994)

[4] V. Devanathan, "Deep inelastic scattering and nucleon structure functions”, in New Perspec- tives in Classical and Quantum Physics, Eds. P.P. Delsanto and A. W. Sáenz, Gordon and Breach, London (1995)

[5] V. Devanathan, Parton spin distribution functions, in Selected Topics in Mathematical Physics: Professor R. Vasudevan Memorial Volume, Eds. R. Sridhar, K. Srinivasa Rao and V. Lakshminarayanan, Allied Publishers Ltd. (1995)

[6] V. Devanathan and J. S. McCarthy, Mod. Phys. Lett. A11, 147 (1996)

[7] V. Devanathan, Ch. 14, Nuclear Physics, Narosa Publishing House, New Delhi, India and Alpha Science International, Oxford, UK. (2006)

[8] M. Klein, structure functions in deep inelastic scattering, 482(1999). 